\title{
Diffusion Coefficients in Aqueous Solutions of Potassium Sulfocyanate at 298.15 K
}

\author{
Ana C.F. Ribeiro, Victor M.M. Lobo and Joaquim J.S. Natividade \\ Department of Chemistry, University of Coimbra \\ 3004 - 535 Coimbra, Portugal \\ e-mail: <vlobo@ci.uc.pt>
}

Received 25 July 2000; accepted 8 February 2001

Key Words: Diffusion / Electrolytes / Aqueous Solutions / Transport Properties

\begin{abstract}
Differential diffusion coefficients of potassium sulfocyanate in water at $298.15 \mathrm{~K}$, and at concentrations from $0.001 \mathrm{M}$ to $1 \mathrm{M}$, have been measured using a conductimetric cell and an automatic apparatus to follow diffusion. The results are discussed on the basis of the OnsagerFuoss and Pikal models. The cell uses an open-ended capillary method and a conductimetric technique is used to follow the diffusion process by measuring the resistance of a solution inside the capillaries, at recorded times.

(C) 2001 Elsevier Science B.V. All rights reserved.
\end{abstract}

\section{Introduction}

Data on diffusion coefficients of potassium sulfocyanate in aqueous solutions are reported [1] only at $25^{\circ} \mathrm{C}$ and only from 0.0128 to $10 \mathrm{M}$. The present paper reports the experimental data for differential diffusion coefficients of potassium sulfocyanate at $298.15 \mathrm{~K}$ but in dilute solutions, i.e., from $0.001 \mathrm{M}$ to $1 \mathrm{M}$, obtained by the open-ended capillary cell previously reported [2]. These results are discussed on the basis of the Onsager-Fuoss [3, 4] and Pikal $[4,5]$ models. 


\section{Experimental}

The apparatus assembled for use with the open-ended capillary cell in this laboratory is essentially the same as previously reported [2]. The cell has two vertical capillaries, each closed at one end by a platinum electrode and positioned one above the other with the open ends separated by a distance of about $10 \mathrm{~mm}$.

The upper and lower tubes, initially filled with solutions of concentrations $0.75 c$ and $1.25 c$, respectively, are surrounded with a solution of concentration $c$. This ambient solution is contained in a glass tank $200 \times 140 \times 60 \mathrm{~mm}$ immersed in a thermostat at $298.15 \mathrm{~K}$. The tank is divided internally by Perspex sheets and a glass stirrer creates a slow lateral flow of ambient solution across the open ends of the capillaries. Experimental conditions are such that the concentration at each of the open ends is equal to the ambient solution value $c$, that is the physical length of the capillary tube coincides with the diffusion path or, in other words, the boundary conditions described in [2] to solve Fick's second law of diffusion are applicable. Therefore, the so-called $\Delta$ l-effect [2] is reduced to negligible proportions. In a manual apparatus, diffusion is followed by measuring the ratio of resistances of upper and lower tubes by an alternating current transformer bridge. In an automatic apparatus $w=R_{t} / R_{b}$ is measured by a Solartron digital voltmeter (DVM) 7061 with 6 1/2 digits. A power source Bradley Electronics Model 232 supplies a $30 \mathrm{~V}$ sinusoidal signal of $4 \mathrm{kHz}$ (stable up to $0.1 \mathrm{mV}$ ) to a potential divider that applies a $250 \mathrm{mV}$ signal to the platinum electrodes at the top and bottom capillaries. By measuring the voltages $\mathrm{V}$ ' and $\mathrm{V}$ " from top and bottom electrodes to the central electrode at ground potential, in a fraction of a second, the DVM calculates $w=R_{t} / R_{b}$. In order to measure the differential diffusion coefficient $D$ at a given concentration $c$, a "top" solution of concentration $0.75 c$ and a "bottom" solution $1.25 c$ are prepared, each in a 2 litre volumetric flask. The "bulk" solution of concentration $c$ is prepared by mixing 1 litre of "top" solution with 1 litre of "bottom" solution, accurately measured. The potassium sulfocyanate solutions were prepared from pro analysi Merck reagent. The glass tank and the two capillaries are filled with $c$ solution, immersed in the thermostat, and allowed to come to thermal equilibrium. TRinf $=10^{4} /(1+w)$, where $w=R_{t} / R_{b}$ is the electrical resistance $(R)$ ratio of the top (t) and bottom (b) diffusion capillaries at infinite time (when their solutions are c), is now measured very accurately. TR $=10^{4} /(1+\mathrm{w})$, is the equivalent, at any time $\mathrm{t}$. 
The capillaries are then filled with "top" and "bottom" solutions and allowed to diffuse into the "bulk" solution. Resistance ratio readings are taken at recorded times, beginning 1000 min after the starting of an experiment. The diffusion coefficient is finally evaluated using a linear least-squares procedure to fit the data and subsequently an itterative process by using 20 terms of the expansion series of the solution of Fick's $2^{\text {nd }}$ law for the present boundary conditions. The theory developed for this cell has already been described [2].

\section{Results and discussion}

The results of diffusion experiments in solutions $0.1 \mathrm{M} \mathrm{KSCN}$ at $298.15 \mathrm{~K}$ are shown in Table 1. They are in good agreement and indicate the precision of the method for measuring differential diffusion coefficients. Measurements in $0.1 \mathrm{M} \mathrm{KCl}$ solutions by this method disagree with those of Miller within 1\% [6]. Table 2 shows the results with potassium sulfocyanate solutions from $1 \times 10^{-3} \mathrm{M}$ to $1 \mathrm{M}$ at $298.15 \mathrm{~K}$. These results are the average of 4 experiments performed in consecutive days. The reproducibility of the results is good, as shown by $\mathrm{S}_{\mathrm{Dav}}$, and its mean is in good agreement with experimental results by Mitchell et al. [1], as shown in Fig. 1 and Fig.2.

\section{Table 1}

Diffusion experiments with $0.1 \mathrm{M} \mathrm{KSCN}$ in aqueous solutions at $298.15 \mathrm{~K}$ using a $4000 \mathrm{~Hz}$ signal

\begin{tabular}{|cc|}
\hline $\mathbf{D} /$ \\
$\mathbf{1 0}^{-\mathbf{9}} \mathbf{~}^{\mathbf{2}} \mathbf{~ s}^{\mathbf{- 1}}$ & TRinf \\
\hline 1.746 & 5005.0 \\
1.738 & 5005.7 \\
1.747 & 5005.4 \\
1.741 & 5005.6 \\
\hline
\end{tabular}

$\mathrm{D}_{\mathrm{av}}=1.743 \times 10^{-9} \mathrm{~m}^{2} \mathrm{~s}^{-1} ; \mathrm{S}_{\mathrm{Dav}}=0.002 \times 10^{-9} \mathrm{~m}^{2} \mathrm{~s}^{-1} ; \mathrm{Cv}=0.2 \%$ where $D_{a v}$ is the mean diffusion coefficient of 4 experiments; $S_{D a v}$ is the standard deviation of that mean and $\mathrm{Cv}$ is its coefficient of variation. 
Table 2

Diffusion coefficients, D, of KSCN in aqueous solutions at $298.15 \mathrm{~K}$ at different concentrations, c, using a $4000 \mathrm{~Hz}$ signal

\begin{tabular}{|c|c|c|c|}
\hline $\begin{array}{c}\mathrm{c} / \\
/ \mathrm{mol} \mathrm{dm}^{-3}\end{array}$ & $\begin{array}{c}\text { Mean value of } D \text { in } \\
4 \text { experiments/ } \\
/ 10^{-9} \mathrm{~m}^{2} \mathrm{~s}^{-1}\end{array}$ & $\begin{array}{l}\text { TRinf } \\
\text { a) }\end{array}$ & $\begin{array}{c}S_{\text {Dav }} \\
/ 10^{-9} \mathrm{~m}^{2} \mathrm{~s}^{-1} \\
\text { b) }\end{array}$ \\
\hline 0.001 & 1.800 & 44990.0 & 0.015 \\
\hline 0.005 & 1.756 & 4993.6 & 0.017 \\
\hline 0.008 & 1.746 & 4997.0 & 0.014 \\
\hline 0.010 & 1.736 & 4998.2 & 0.016 \\
\hline 0.020 & 1.725 & 5003.5 & 0.008 \\
\hline 0.030 & 1.714 & 5005.0 & 0.008 \\
\hline 0.050 & 1.739 & 5006.0 & 0.011 \\
\hline 0.080 & 1.764 & 5008.0 & 0.009 \\
\hline 0.100 & 1.743 & 5005.4 & 0.002 \\
\hline 0.200 & 1.748 & 5005.0 & 0.006 \\
\hline 0.500 & 1.734 & 4990.5 & 0.008 \\
\hline 1.000 & 1.740 & 4996.0 & 0.005 \\
\hline
\end{tabular}

a) TRinf $=10^{4} /(1+w)$, where $w=R_{t} / R_{b}$ is the resistance ratio. b) $S_{D a v}$ is the standard deviation of the mean of 4 experiments.

Fig.1 and Fig.2 show a good agreement between our experimental results (Table 2) and those obtained by Mitchell et al. [1]. This agreement is within $98.0 \%$. Our equations of adjustment (eq. 1 and eq.2) are:

$$
\begin{gathered}
\mathrm{D} /\left(10^{-9} \mathrm{~m}^{2} \mathrm{~s}^{-1}\right)=1.857-1.892 \sqrt{\mathrm{c}}+7.374 \mathrm{c}-5.833 \mathrm{c}^{3 / 2} \\
\left(\mathrm{c} \leq 5 \times 10^{-2} \mathrm{~mol} \mathrm{dm} \mathrm{d}^{-3}\right) \\
\mathrm{D} /\left(10^{-9} \mathrm{~m}^{2} \mathrm{~s}^{-1}\right)=1.855-0.6758 \sqrt{\mathrm{c}}+1.155 \mathrm{c}-0.5959 \mathrm{c}^{3 / 2} \\
\left(\mathrm{c} \geq 5 \times 10^{-2} \mathrm{~mol} \mathrm{dm}^{-3}\right)
\end{gathered}
$$




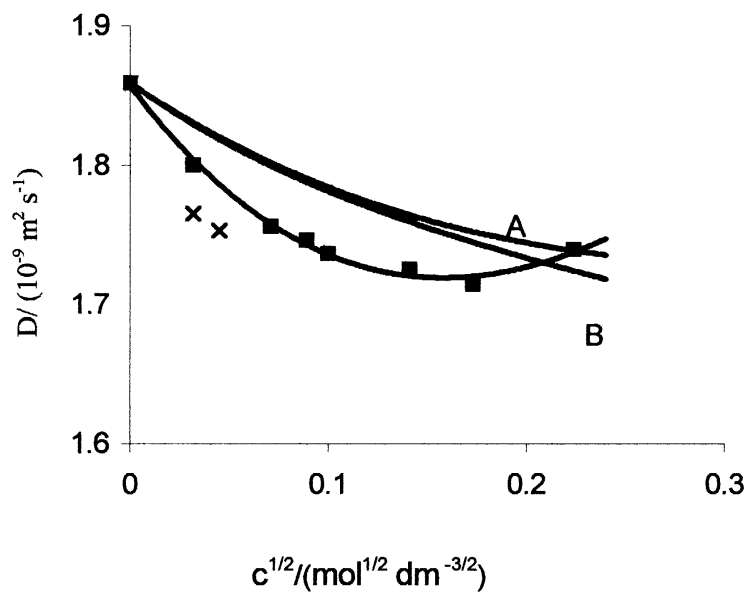

Figure 1

Comparison of diffusion coefficients of $\mathrm{KSCN}-\mathrm{H}_{2} \mathrm{O}$ with Onsager-Fuoss and Pikal models (curves A and $\mathrm{B}$, respectively), for dilute solutions, using Kielland 's value $\left(\mathrm{a}=3.2 \times 10^{-10} \mathrm{~m}\right)$ for the ion size parameter, a, and our results $(\varpi)$, at $298.15 \mathrm{~K}$. Other experimental results by Mitchell et al. (x)

Fig.1 and Fig.2 compare experimental results with calculations on the basis of Onsager-Fuoss $[3,4]$ and Pikal $[4,5]$ models. For dilute solutions the experimental data and both models are in reasonable agreement; for higher concentrations, our experimental data and those from other reseachers are in good agreement, but the results from the above models differ considerably from experimental observation. This is not surprising if we take into account the change with concentration of parameters such as viscosity, dielectric constant and hydration, factors not taken into account in Onsager-Fuoss and Pikal models.

Pikal developed a theory of mutual diffusion in binary electrolytes using the conventional charged sphere-in-continuum model. However, instead of approximating the Boltzmann exponential by a truncated power series, the calculations are performed retaining the full Boltzmann exponential. As a result of this procedure, a term representing the effect of ion-pair formation appears in the theory as a natural consequence of the electrostatic interactions. However, the results obtained by this equation in diluted solutions are similar to 
Onsager's results. We may therefore admit, that the effects of short range interactions on the diffusion of this electrolyte at those concentrations are not relevant.

Also, the relative viscosity factor [7] seems to have a negligible role on the diffusion of this salt: it does not significantly affect diffusion calculations with Onsager-Fuoss [3, 4] or Pikal $[4,5]$ models. Admitting hydration of the $\mathrm{K}^{+}$, with, e.g., $\mathrm{h}=1$ [8], we obtain results from Agar's equation [8] similar to those from the above sources [3-5].

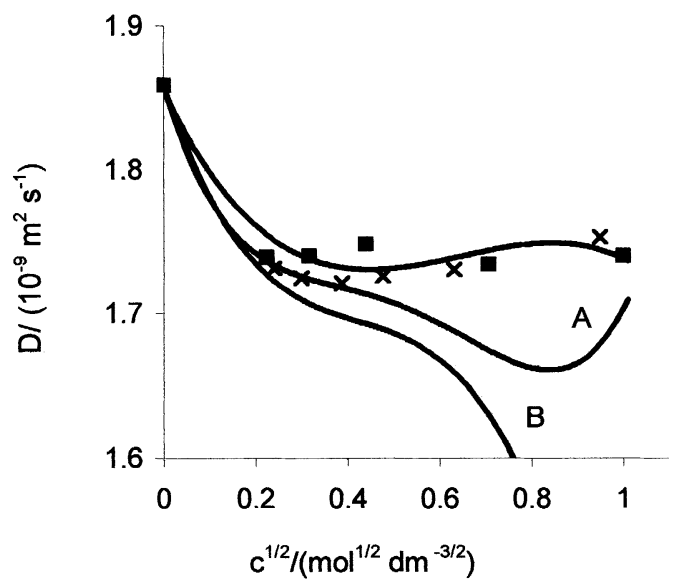

Figure 2

Comparison of diffusion coefficients of $\mathrm{KSCN}-\mathrm{H}_{2} \mathrm{O}$ with Onsager-Fuoss and Pikal models (curves A and B, respectively), for concentrated solutions, using Kielland ' $\mathrm{s}$ value $\left(\mathrm{a}=3.2 \times 10^{-10} \mathrm{~m}\right)$ for the ion size parameter, a, and our results ( $\square$ ), at $298.15 \mathrm{~K}$. Other experimental results by Mitchell et al. (x)

\section{References}

[1] J.P. Mitchell, J.B. Butler and J.G. Albright, J. Sol. Chem. 21, 1115 (1992).

[2] V.M.M. Lobo, Ph.D. Thesis, Cambridge (1971); J.N. Agar and V.M.M. Lobo, J. Chem. Soc., Faraday Trans., I 71, 1659 (1975).

[3] L. Onsager and R.M. Fuoss, J. Phys. Chem. 36, 2689 (1932).

[4] V.M.M. Lobo, A.C.F. Ribeiro and S.G.C.S. Andrade, Ber. Bunsenges. Phys. Chem. 99, 713 (1995).

[5] M.J. Pikal, J. Phys. Chem. 75, 663 (1971).

[6] V.M.M. Lobo, A.C.F. Ribeiro and L.M.P. Verissimo, J. Mol. Liquids 78, 139 (1998).

[7] A.R. Gordon, J. Chem. Phys. 5, 522 (1937).

[8] R. A. Robinson and R.H. Stokes (Electrolyte Solutions), $2^{\text {nd }}$ Ed., Butterworths, London (1959). 